\title{
Magnetic Field Simulation of Golay Coil
}

\author{
Rashdi Shah Ahmad, Amiruddin Bin Shaari, Chew Teong Han * \\ Department of Physics, Faculty of Science, Universiti Teknologi Malaysia, 81310 UTM Skudai, Johor, Malaysia. \\ * Author to whom correspondence should be addressed; E-mail: rashdishah@yahoo.com, amiryeng@yahoo.com and \\ teonghan@yahoo.com
}

Received: 30 October 2008

http://dx.doi.org/10.11113/mjfas.v4n2.43

\section{ABSTRACT}

In the field of magnetic resonance imaging (MRI), besides the main magnetic field and the radio frequency (RF) system, the gradient coils system also plays an important role in signal acquisition. Generally, there are 2 transverse gradient coils and 1 longitudinal gradient coil. In this research, the magnetic field (or gradient) generated by the conventional transverse coil, namely the Golay coil was mapped. The calculation algorithm of the magnetic field generated was written in C-programming language, compiled by the GNU-compiler collection (GCC) and was based on a forward analytical approach by using the Biot-Savart Law. The data was then visualized by Gnuplot. The magnetic field was successfully calculated and plotted using open-source softwares running under Ubuntu operating system and will provide valuable data especially for gradient coil designer.

| Magnetic resonance imaging (MRI) | Golay coil | simulation | GCC | Gnuplot |

\section{Introduction}

Gradient coils play an important role in magnetic resonance imaging (MRI) since they directly affect the resolution of the signal. There are usually three sets of gradient coils in a MRI system, each generating a magnetic gradient along each orthogonal axis, namely $x, y$ and $z$. The longitudinal gradient coil will generate a magnetic gradient along $z$-direction which is derived from the shape of Maxwell coil. The two other transverse gradient coils however consist of double saddle configuration, known as the Golay coil [1], made up from two saddle coils. The main purpose of gradient coils in a MRI system is to produce a magnetic gradient along each axis, helping in slice selection, frequency encoding and phase encoding. Technically speaking, the gradient would be preferred to be as linear as possible over a large volume. However, due to several constraints such as the inductance and the size of the coil, these few characteristics will have to be traded off with each other.

\section{Methodology}

The magnetic field generated by a finite current segment can be calculated using an analytical approach by using the Biot-Savart Law [2]. Any coil shape can be divided into a series of finite current segment and by using the Biot-Savart Law for finite current segment, the magnetic field generated by the coil at any point can be 
calculated. Equation 1 shows the Biot-Savart Law for a finite current segment [3] with Figure 1 showing the related parameters.

$$
B=\frac{\mu_{0} I}{4 \pi a}\left(\cos \theta_{1}-\cos \theta_{2}\right)
$$

From Figure 1, the magnetic field induced by the finite segment $\overrightarrow{A B}$ at point $M$ can be calculated using Equation 1. However, before doing any calculation, further vector analysis is required to obtain all the necessary parameters.

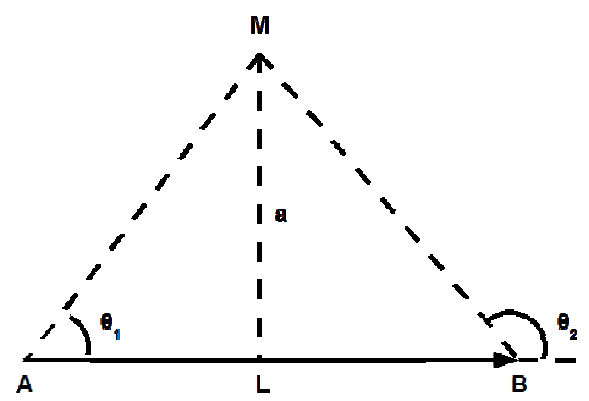

Figure 1: Parameters Related to Finite Segment with Calculation Point, $M$

The direction of $\vec{B}$ is obtained by taking the cross product of the unit vector of $\overrightarrow{A B}$ and the unit vector along a line joining the current segment to the calculation point, $M(\overrightarrow{L M})$. The vector of the current segment, $\overrightarrow{A B}$ and its unit vector, $\hat{u}_{A B}$, are given by Equation 2 and 3 :

$$
\begin{gathered}
\overrightarrow{A B}=\overrightarrow{O B}-\overrightarrow{O A} \\
\hat{u}_{A B}=\frac{\overrightarrow{A B}}{|\overrightarrow{A B}|}
\end{gathered}
$$

Similarly, $\overrightarrow{A M}$ and $\overrightarrow{B M}$ as well as their respective unit vectors are given by Equation 4 to 7 :

$$
\begin{gathered}
\overrightarrow{A M}=\overrightarrow{O M}-\overrightarrow{O A} \\
\overrightarrow{B M}=\overrightarrow{O M}-\overrightarrow{O B} \\
\hat{u}_{A M}=\frac{\overrightarrow{A M}}{|\overrightarrow{A M}|} \\
\hat{u}_{B M}=\frac{\overrightarrow{B M}}{|\overrightarrow{B M}|}
\end{gathered}
$$


The cosine of the angles $\theta_{1}$ and $\theta_{2}$ are calculated from Equation 8 and 9:

$$
\begin{aligned}
& \cos \theta_{1}=\hat{u}_{A B} \bullet \hat{u}_{A M} \\
& \cos \theta_{2}=\hat{u}_{A B} \bullet \hat{u}_{B M}
\end{aligned}
$$

The distance between the calculation point, $M$ to the segment is given by the magnitude of $\overrightarrow{L M}$ in Equation 10:

$$
\overrightarrow{L M}=\overrightarrow{A M}-\overrightarrow{A L}
$$

$\overrightarrow{A L}$ is the projection of $\overrightarrow{A M}$ in the direction of $\hat{u}_{A B}$, given by Equation 12 :

$$
\overrightarrow{A L}=\left(\overrightarrow{A M} \bullet \hat{u}_{A B}\right) \hat{u}_{A B}
$$

Therefore,

$$
\overrightarrow{L M}=\overrightarrow{A M}-\left(\overrightarrow{A M} \bullet \hat{u}_{A B}\right) \hat{u}_{A B}
$$

The direction of the magnetic field at the calculation point, $M$ is shown in Equation 13:

$$
\hat{u}_{B}=\hat{u}_{A B} \times \hat{u}_{L M}
$$

Finally, the magnetic field at point $M$ is obtained as:

$$
\vec{B}=\frac{\mu_{0} I}{4 \pi|\overrightarrow{L M}|}\left(\cos \theta_{1}-\cos \theta_{2}\right) \hat{u}_{B}
$$

Figure 2 shows the calculation model used in this simulation. The arrows denote the current flow in the Golay coil. The calculations are done on the $x y$-plane, divided in grids, shown in Figure 2.

The calculation grid for both $x y$-plane and $x z$-plane will be the size of $(0.4 \mathrm{~m} \mathrm{X} 0.4 \mathrm{~m})$ with a step of $0.01 \mathrm{~m}$. A total of five coil parameters will be varied and simulated accordingly, namely the separation between two saddle coils, $\boldsymbol{a}$, the length of Golay coil, $\boldsymbol{b}$ (as shown in Figure 2), the arc angle of the coil, $\boldsymbol{\theta}$, the radius of the coil, $\boldsymbol{r}$ and the separation between each half of saddle coil, $\boldsymbol{c}$ (as shown in Figure 3). For each simulation, only one parameter will be varied while the rest will be fixed at certain value. The $\mathrm{C}$-code of the program would iterate the calculation and export the data into a text file which was then visualized by Gnuplot.

\section{Results and Discussions}

Figure 4 to 9 show the some of the results obtained by the simulation. Figure 4 to 6 show the magnetic contour plot of the results to show how uniform is the gradient generated by altering one of the coil geometry 
mentioned above. Figure 7 to 9 show the normal xy-plot of the same results to show how linear is the gradient, the gradient value itself and also the gradient uniformity. Figure 4 to 9 were plotted for an $x$-range with different values of $y$. The graphs were then plotted on the same axis, overlapping each other. The idea was that for different values of $y$, if the xy-plots were perfectly overlapped, and then one can consider the gradient was very uniform along the $y$-direction.

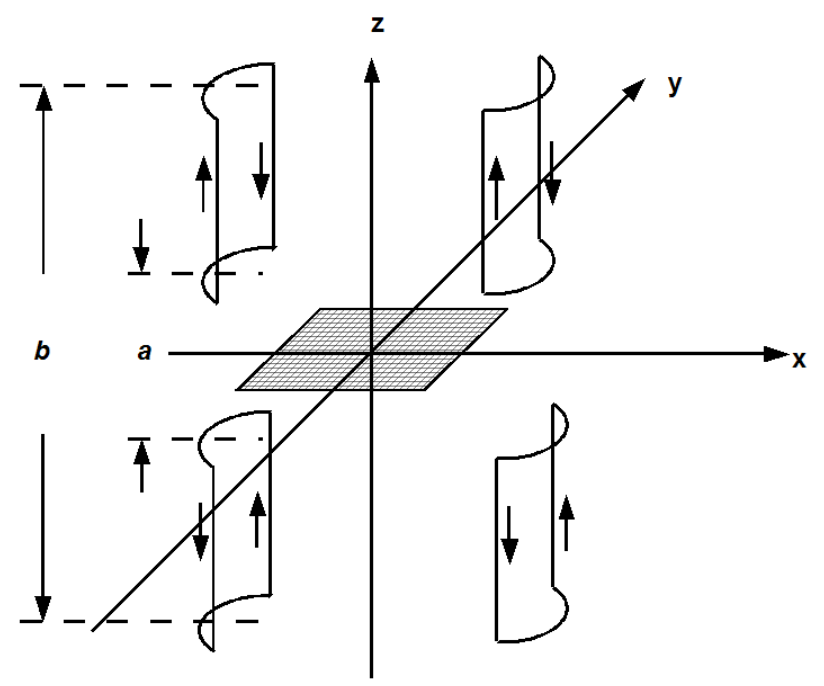

Figure 2: Calculation Model

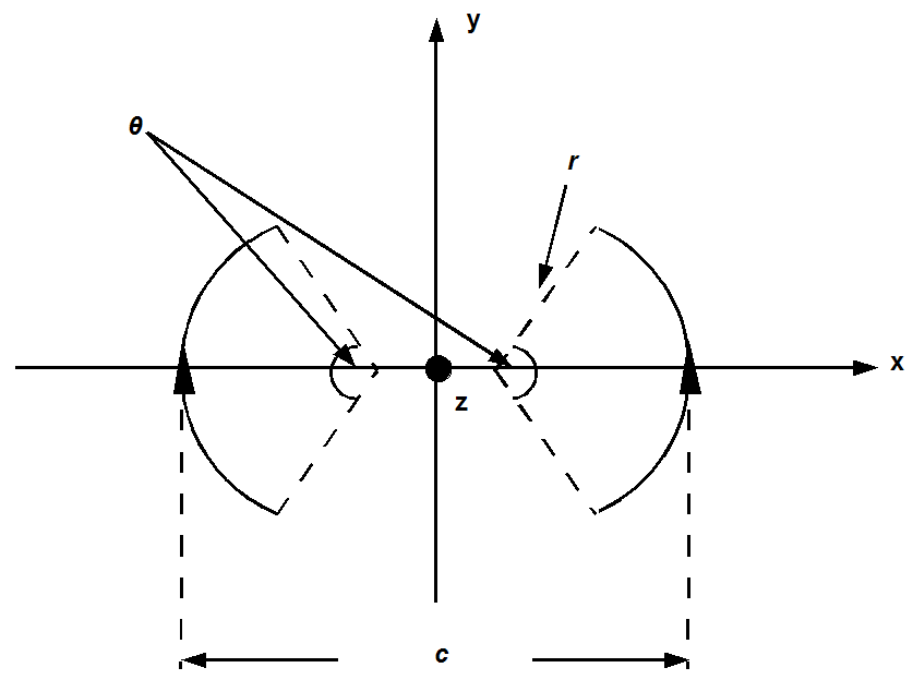

Figure 3: Top View of Calculation Model with $z$-axis pointing out

From Figure 4 and 7, as $\boldsymbol{a}$ increases, the gradient uniformity decreases, the gradient linearity increases whereas the gradient value decreases. From Figure 5 and 8, as $\boldsymbol{b}$ increases, the gradient uniformity slightly decreases almost no change in gradient linearity whereas the gradient value slightly changes. From Figure 6 and 
9, as $c$ increases, the gradient uniformity decreases, almost no change in gradient linearity whereas the gradient value decreases.
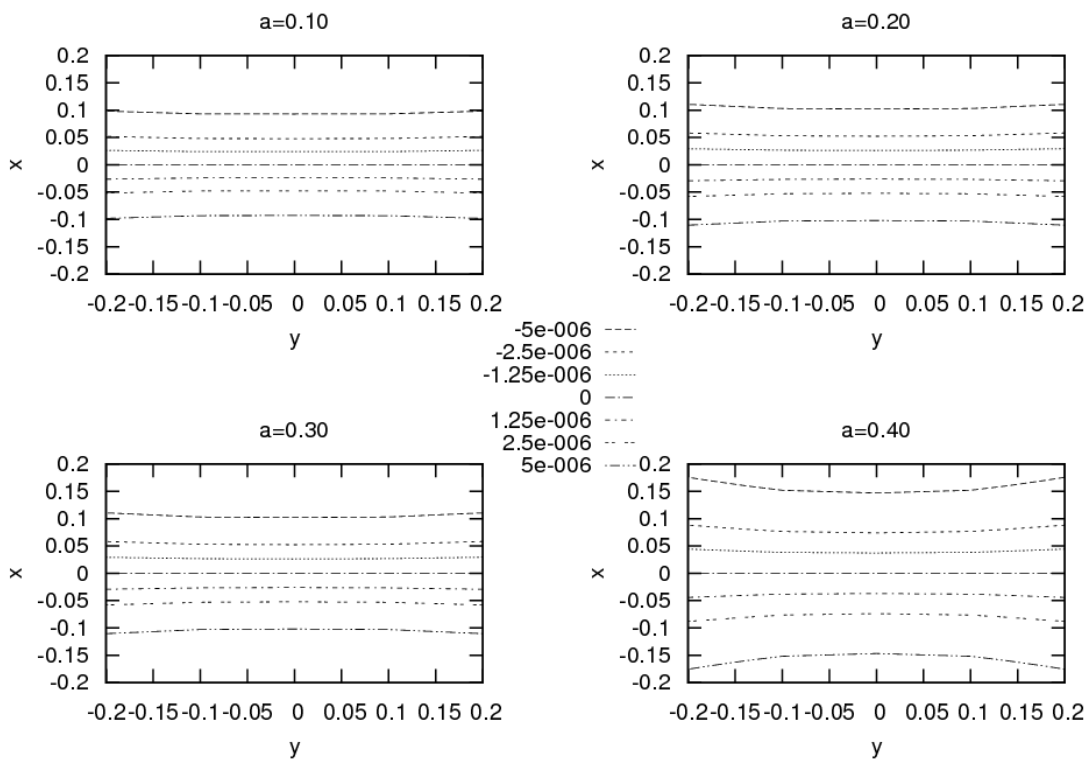

Figure 4: Magnetic Contour Plot for Various $\boldsymbol{a}$
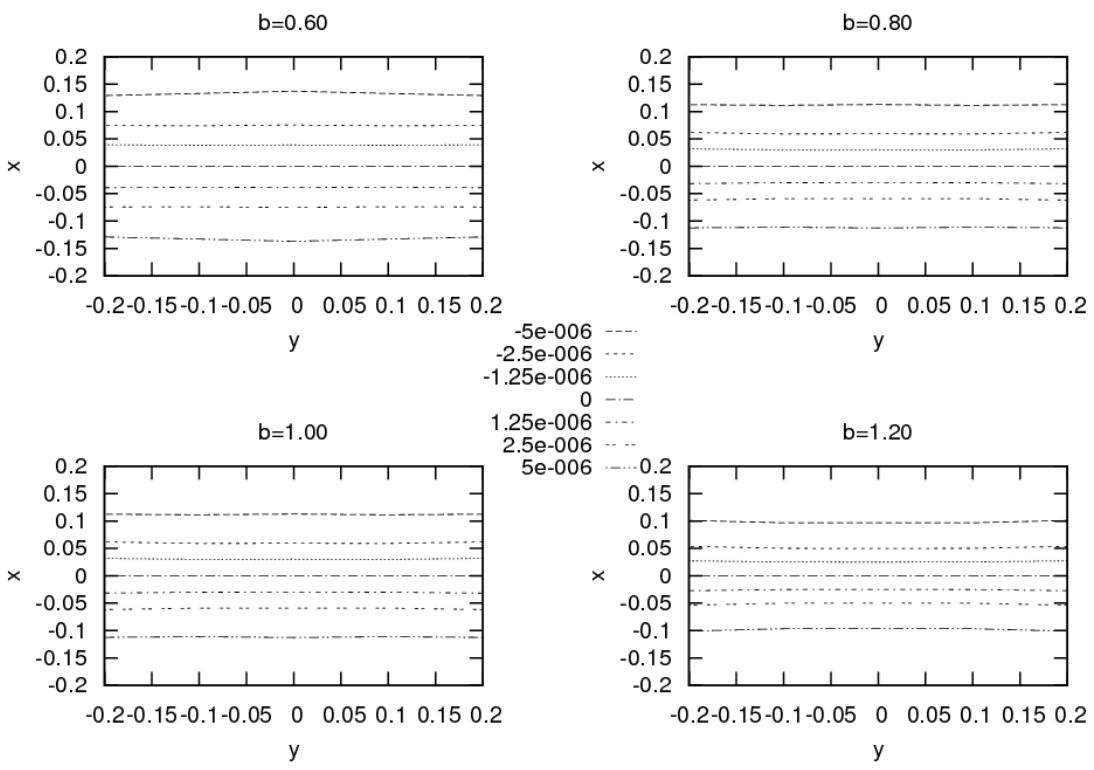

Figure 5: Magnetic Contour Plot for Various $\boldsymbol{b}$ 


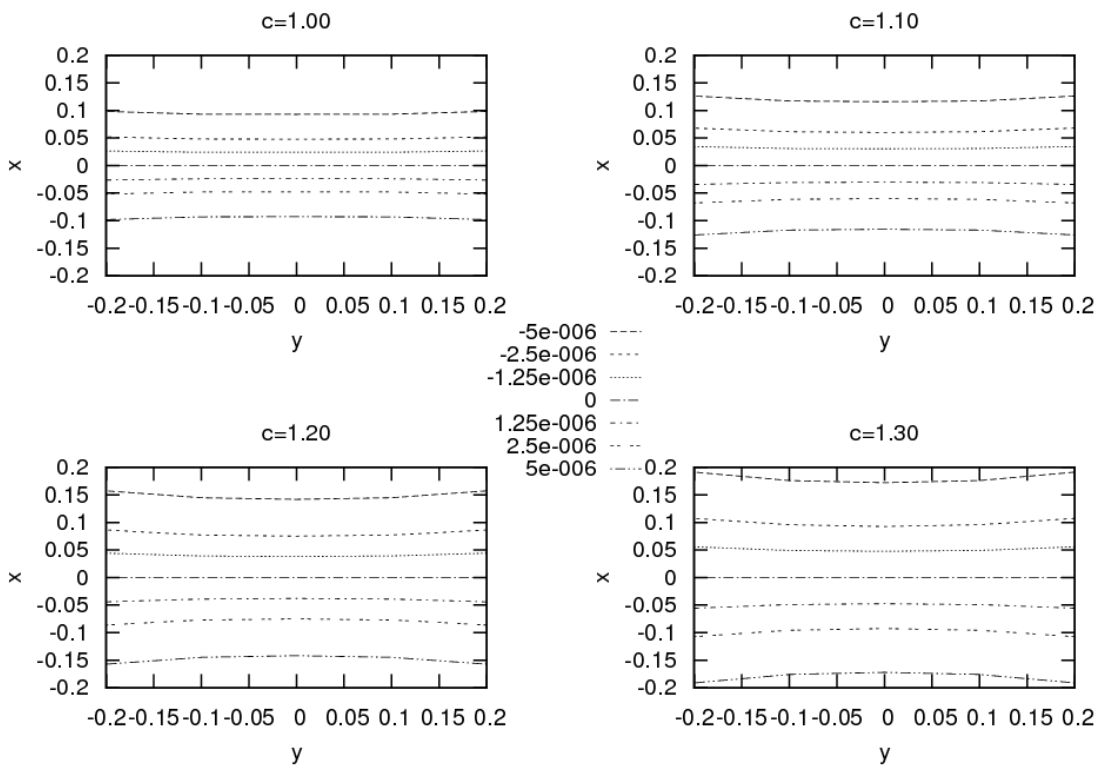

Figure 6: Magnetic Contour Plot for Various $c$
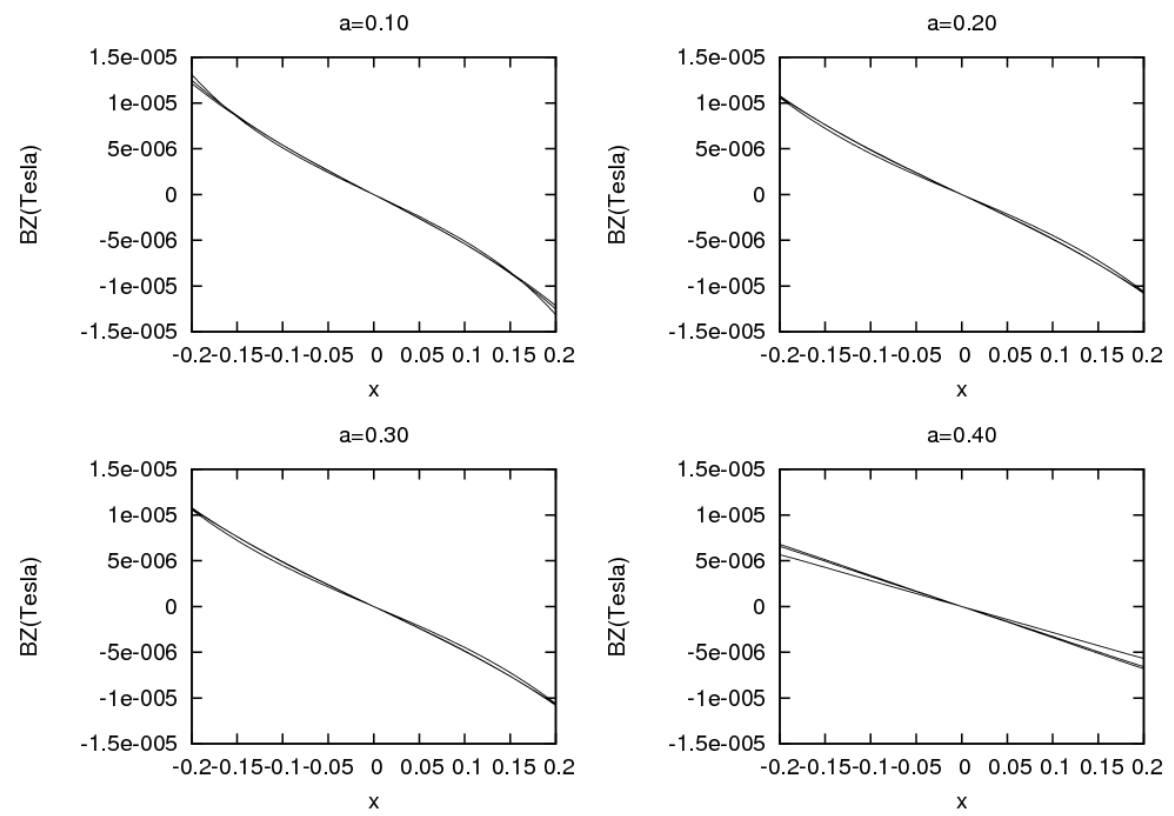

Figure 7: xy-plot for various $\boldsymbol{a}$ 

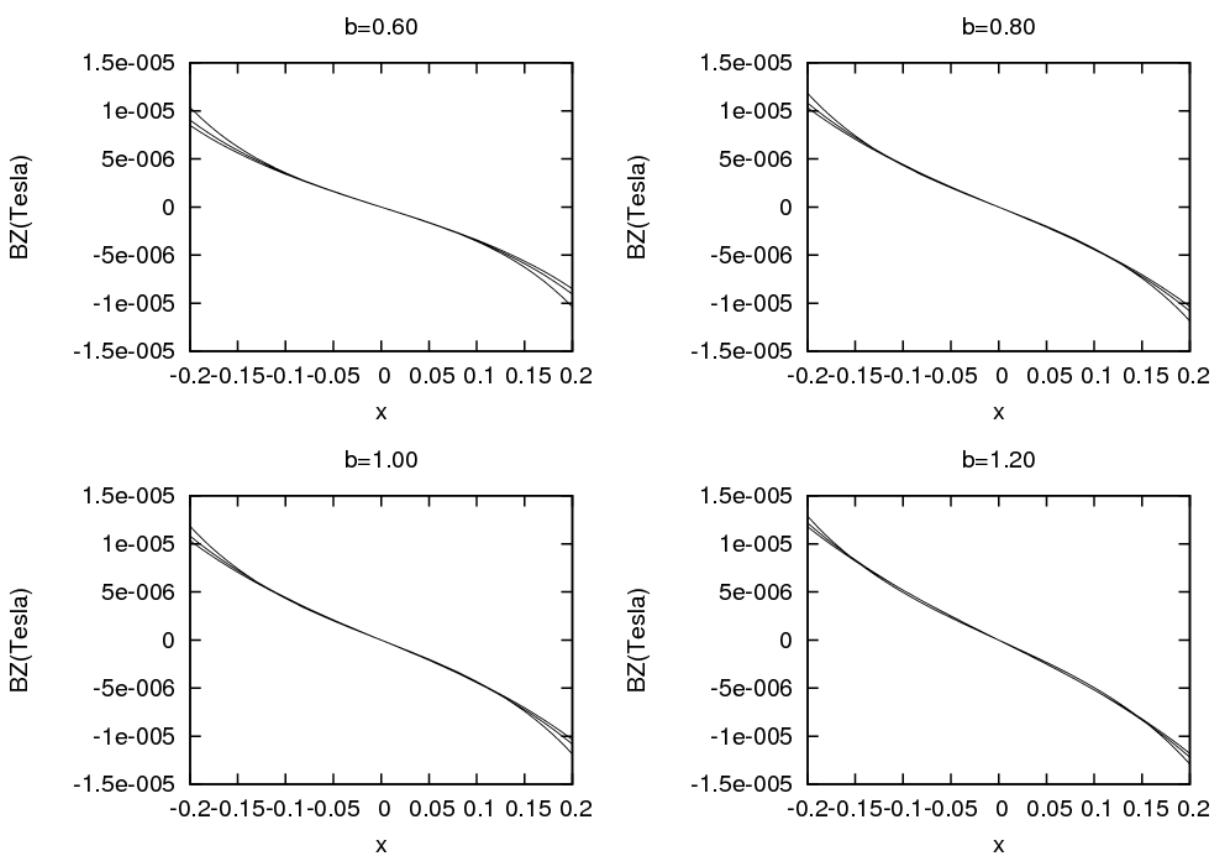

Figure 8: xy-plot for various $\boldsymbol{b}$
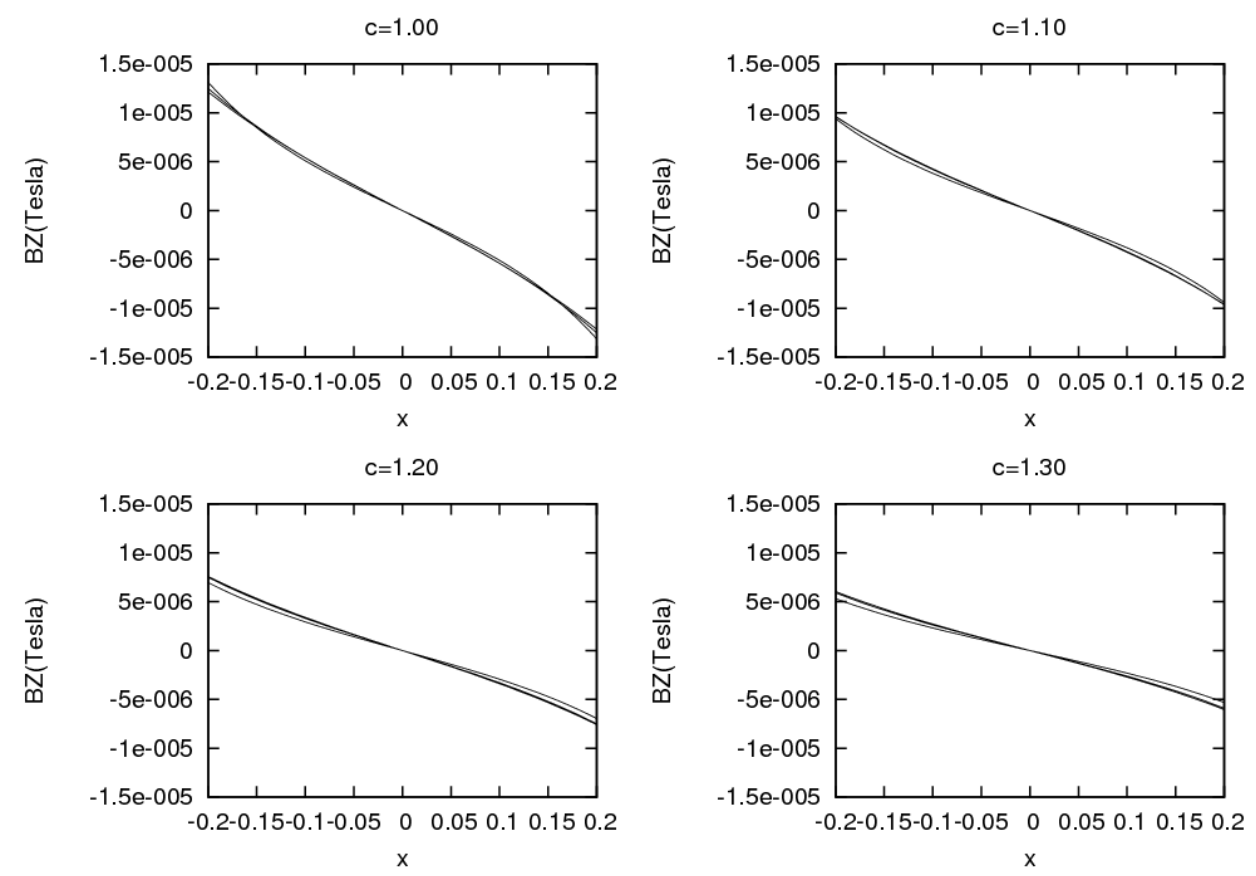

Figure 9: xy-plot for various $c$ 
Other results obtained by varying the $\boldsymbol{r}$ parameter and the $\boldsymbol{\theta}$ parameter. As $\boldsymbol{r}$ increases, almost no change in gradient uniformity, gradient linearity increases whereas the gradient value decreases. As expected, $\boldsymbol{\theta}=120^{\circ}$ offered the best gradient uniformity among the values of $\boldsymbol{\theta}$. Figure 10 and 11 show the ideal data plot generated. Analytically speaking, if the magnetic field mapping obtained, both experimentally or theoretically (through computer simulation), is as similar to these plots, the better the gradient coil is, in terms of both field linearity and gradient uniformity.

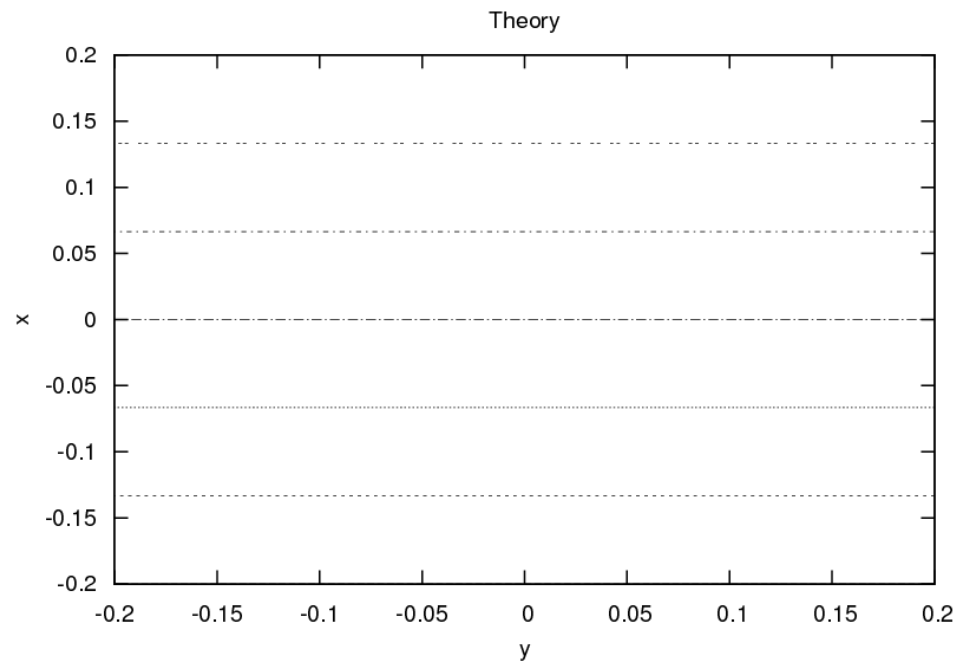

Figure 10: Ideal Contour Plot for x-Golay Coil

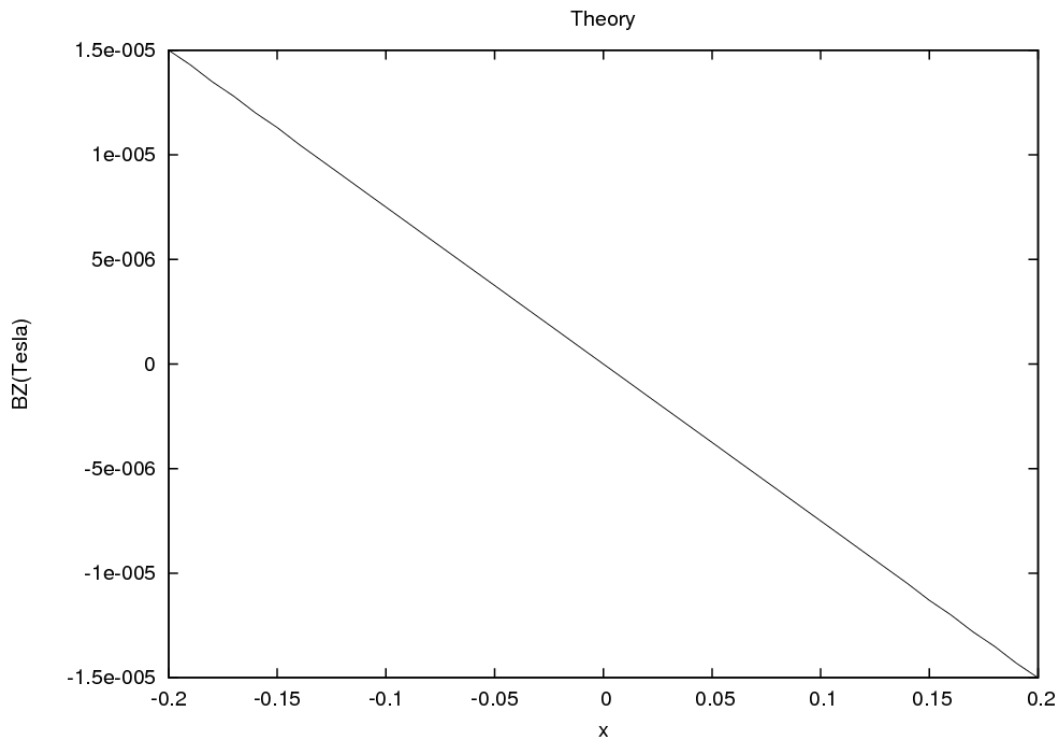

Figure 11: Ideal xy-plot for x-Golay Coil 


\section{Conclusion}

The simulation is considered success with few rooms for improvement including numerical analysis for more accurate gradient calculations, mapping for magnetic gradient values rather than the magnetic field and etc. The simulation also proves to be a great tool and to provide assistance especially to gradient coil designers.

\section{References and Citations}

[1] Adamiak, K., Rutt, B. K., and Dabrowki, W. J., Design of Gradient Coils for Magnetic Resonance Imaging, IEEE Transactions on Magnetics, Vol 28, pg 2403-2405, 1992

[2] Rashdi Shah Ahmad and Chew, T. H., Algorithm for Magnetic Field Visualization of a Flat Plane Induced by Finite Dipole Segment Using GCC and Gnuplot, Regional Annual Fundamental Science Seminar, 2007

[3] Sadiku, M., 1995. Elements of Electromagnets. Oxford University Press. 